\title{
Contributions of the loops on the stability and targeting of DNA pseudoknots
}

\author{
Calliste Reiling and Luis A. Marky* \\ *Correspondence: Imarky@unmc.edu

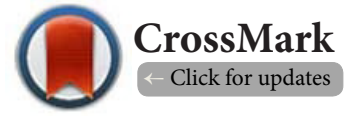

Department of Pharmaceutical Sciences, College of Pharmacy, University of Nebraska Medical Center, 986025 Nebraska Medical Center, Omaha, Nebraska, United States.

\begin{abstract}
Background: Pseudoknots have been found to play important roles in RNA function, examples include ribosomal frameshifting and in the 5' UTR of mRNA as riboswitches. In RNA frameshifting, there is a local formation of base-triplet stacks within the pseudoknot, increasing the stability of the terminal stem. The interaction of this triplex structure with the ribosome might help with the high-efficiency of frameshifting. The main objective of this work is to mimic RNA pseudoknots using DNA oligonucleotides for the control of gene expression. Specifically, we have designed a pair of DNA pseudoknots with different length in one of the loops to mimic the formation of a local triple helix, shown within RNA pseudoknots of the human telomerase.

Methods: We have used a combination of temperature-dependent UV spectroscopy and calorimetric techniques to determine the thermodynamics for the unfolding of the pseudoknots and their targeting with complementary strands. The unfolding data is then used to create thermodynamic (Hess) cycles that correspond to each of the targeting reactions. The resulting data is then compared with the thermodynamic enthalpy data obtained directly from isothermal titration calorimetry.

Results: UV melting curves of each pseudoknot show transitions with $T_{\mathrm{M}} \mathrm{s}$ independent of strand concentration, which confirms their intramolecular formation. Analysis of the differential scanning calorimetry (DSC) curves shows the pseudoknot with the longer thymine loop (PsK-9) to be more stable, by $-5.7 \mathrm{kcal} / \mathrm{mol}$, and to unfold with a higher enthalpy of $27.4 \mathrm{kcal} / \mathrm{mol}$. The targeting of each pseudoknot yielded favorable reaction free energy contributions that were enthalpy driven. However, the disruption reaction of PsK-9 took place with a less favorable free energy term, by $0.7 \mathrm{kcal} / \mathrm{mol}$, and less favorable enthalpy term, by $4.5 \mathrm{kcal} / \mathrm{mol}$.

Conclusion: The thermodynamic unfolding data showed that PsK-9 is more stable or more compact, due to the involvement of three loop thymines of PsK-9 in forming three T*AT base-triplets, or two T*AT/ T*AT base-triplet stacks, in the stem of this pseudoknot. The targeting thermodynamic data indicated that each complementary strand is able to disrupt the pseudoknots. However, the disruption of PsK-9 takes place with a less favorable free energy contribution, confirming the formation of a short and local triplex.
\end{abstract}

Keywords: Targeting nucleic acids, intramolecular DNA structures, pseudoknots, antisense, thermodynamics, ITC, DSC

\section{Introduction}

The formation of nucleic acids secondary structures, such as hairpin loops, triplexes, G-quadruplexes, pseudoknots, and i-motifs is well documented [1-4] and they have been postulated to be involved in a variety of biological functions [4-14]. Pseudoknots belong to an interesting and diverse RNA structural motif, due to variation in their loop lengths and stems and the types of interactions between them. Pseudoknots have diverse roles in biological function; examples include forming the catalytic core of various ribozymes [15-17], self-splicing introns [18], telomerase [19,20], riboswitches [21], and ribosomal frameshifting $[22,23]$. In ribosomal frameshifting a pseudoknot and slippery sequence are involved to change the reading frame allowing for different mRNAs to be translated which is 
Reiling et al. Biochemical Compounds 2014,

a very common mechanism found in viruses [22]. It has been shown that a local formation of base-triplet stacks within the pseudoknot increases the stability of the terminal stem and the interaction of this triplex structure with the ribosome might help with the high-efficiency of frameshifting [23]. The targeting of pseudoknots with nucleic acid oligonucleotides (ODNs) may stop their biological regulation [24-29].

ODNs, as drugs, present an exquisite selectivity and are able to discriminate targets that differ by a single base and can be used to control the expression of genes. $[5-7,24]$. There are three main approaches for the use of ODNs as modulators of gene expression: the antisense, antigene, and small interfering RNA strategies [24]. In the antisense strategy, an ODN binds to messenger RNA, forming a DNA/ RNA hybrid duplex that inhibits translation by blocking the assembly of the translation machinery or by inducing an RNase $\mathrm{H}$ mediated cleavage of their mRNA target [5]. In the antigene strategy, an ODN binds to the major groove of a DNA duplex, forming a triple helix [30] that inhibits transcription, by competing with the binding of proteins that activate the transcriptional machinery $[\mathbf{7 , 3 1}]$. There are advantages and disadvantages in these two strategies. In the direct targeting of a gene, the antigene strategy offers some advantages over the antisense strategy. First of all, there are only two copies of a particular gene whereas there is a large continuous supply of the mRNA gene transcript. Moreover, blocking the transcription of the gene itself prevents repopulation of the mRNA pool, allowing a more efficient and lasting inhibition of gene expression $[32,33]$. The main disadvantage is that the ODN needs to cross the nuclear membrane and access its DNA target within the densely packed chromatin structure [34]. Common disadvantages of the use of ODNs for targeting purposes are that the oligonucleotide needs to cross lipid membranes; for instance, hydrophilic ODN duplexes do not cross lipid membranes [35], and the fast degradative action of nucleases. These disadvantages can be circumvented by using single strands and by chemically modification of its phosphate or sugar groups. The presence of unpaired nucleobases renders the ODN more hydrophobic, allowing them to interact better with polycationic micelles and/or enabling them to cross the cellular membranes. These polycations can be used as delivery vectors, protecting the ODN from the action of nucleases.

From a thermodynamic point of view, successful control of gene expression depends on the effective binding of a DNA oligonucleotide sequence to its target with tight affinity and specificity. This is provided by using a long sequence of $15-20$ bases in length when targeting genes [5]; strong specificity is conferred by hydrogen bonding in the formation of WatsonCrick and/or Hoogsteen base-pairs, while high affinity is provided by the large negative free energy upon formation of a duplex or triplex products; thereby, competing efficiently with the proteins involved in transcription or translation. In the successful targeting of nucleic acid secondary structures with complementary strands, the strand must be able to invade and disrupt the secondary structure forming a large number of base-pair stacks in the duplex products. Our laboratory is using DNA oligonucleotides to mimic the secondary structures of RNA molecules and their targeting with complementary strands to create a library of thermodynamic targeting data [27-29]. The novelty of this approach is several fold, DNA oligonucleotides are less expensive than RNA oligonucleotides and more stable against hydrolysis, and most important the resulting DNA-DNA thermodynamic data is similar to the DNARNA thermodynamic data [36-38], which is obtained in the targeting of RNA molecules with DNA complementary strands.

In this work, we have designed a pair of DNA pseudoknots with different length in one of the loops to mimic the formation of a local triple helix shown within RNA pseudoknots of the human telomerase, explaining the increase efficiency of ribosomal frameshift. To this end, we have used a combination of UV spectroscopy and calorimetric techniques to determine the thermodynamics for both the unfolding of both DNA pseudoknots and their targeting with complementary strands. The results show that the pseudoknot with the longer loop is more stable and forming a local triplex structure, consistent of two base-triplet stacks, which is confirmed with the lower free energy obtained in the targeting of this pseudoknot.

\section{Methods}

The 5'-3' sequences of oligonucleotides (ODNs) are as

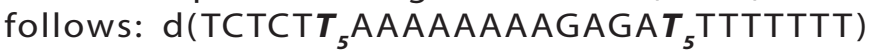

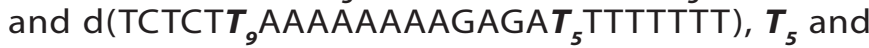
$\boldsymbol{T}_{9}$ are loops of 5 and 9 thymines, respectively; and complementary strands, d(CTTTTTTTTAAAAAAGAGA) and $\mathrm{d}$ (CTTTTTTTTAAAAAAAAAAGAGA). ODNs were synthesized by Integrated DNA Technologies, Inc. (Coralville IA), purified by reverse-phase HPLC, and desalted by gel permeation chromatography using a G-10 Sephadex column. Figure 1 shows the putative secondary structures and designations of pseudoknots, complementary strands and resulting duplexes. The ODN concentrations were determined at $260 \mathrm{~nm}$ and $90^{\circ} \mathrm{C}$ using the following molar extinction coefficients $\left(\mathrm{mM}^{-1} \mathrm{~cm}^{-1}\right)$, obtained from the nearest-neighbor model [39]: 331.1 (PsK-5), 180.6 (CS-5), 255.9 (PsKDup-5), 366.6 (PsK-9), 236.9 (CS-9) and 301.8 (PsKDup-9). All experiments were carried out in $10 \mathrm{mM}$ sodium phosphate $100 \mathrm{mM} \mathrm{NaCl}$ buffer at $\mathrm{pH}$ 7.0. All oligonucleotide solutions were prepared by dissolving dry and desalted ODNs in buffer, and then heating the solution to $70^{\circ} \mathrm{C}$ then cooled to the starting experiment temperature.

\section{Temperature-dependent UV spectroscopy}

Absorbance versus temperature profiles were measured at $260 \mathrm{~nm}$ with a thermoelectrically controlled Aviv Spectrophotometer Model 14DS UV-Vis (Lakewood, NJ). The temperature was scanned at a heating rate of $0.6^{\circ} \mathrm{C} / \mathrm{min}$, and shape analysis of the melting curves yielded transition temperatures, $T_{\mathrm{M}} \mathrm{s}[40]$. The transition molecularity for the 


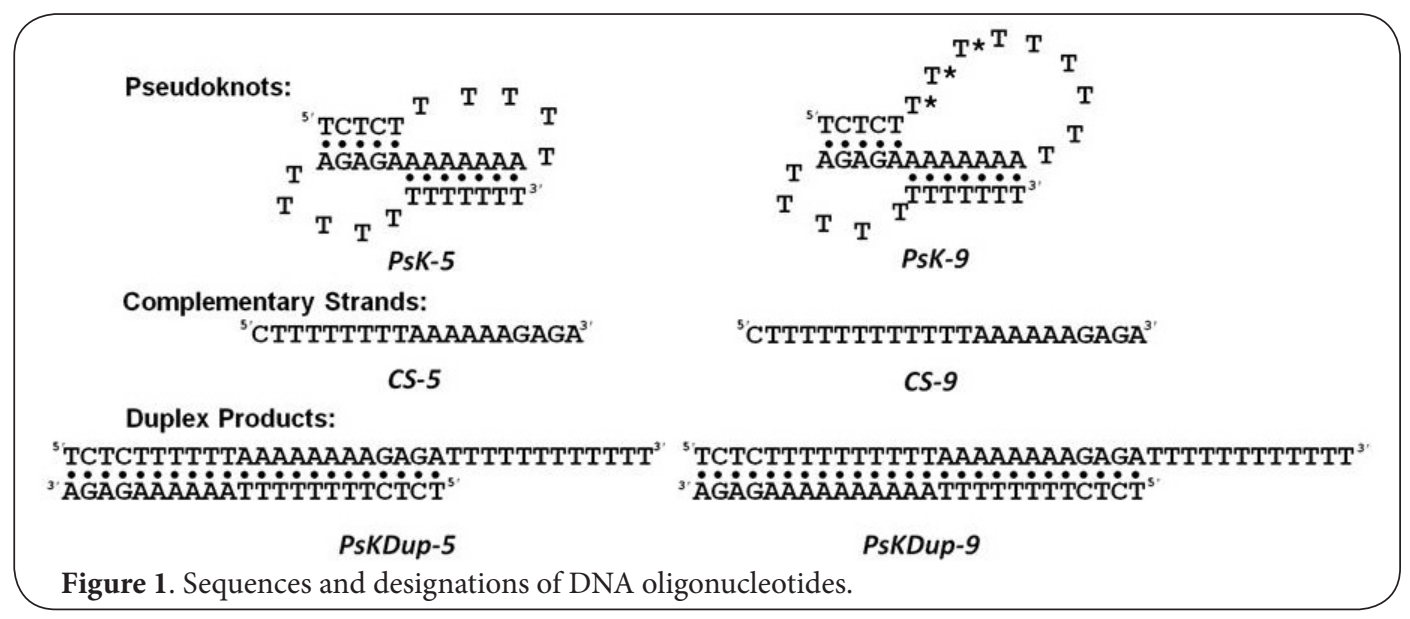

unfolding of a particular complex was obtained by monitoring $T_{M}$ as a function of the strand concentration. Intramolecular complexes show a $T_{M}$-independence on strand concentration, while the $T_{\mathrm{M}}$ of intermolecular complexes does depend on strand concentration [41].

\section{Differential scanning calorimetry (DSC)}

The total heat required for the unfolding of each oligonucleotide (pseudoknot, single strand or duplex product) was measured with a VP-DSC differential scanning calorimeter from Microcal (Northampton, MA). Standard thermodynamic profiles and $T_{M} S$ are obtained from the DSC experiments using the following relationships $[40,41]: \Delta H=\int \Delta C_{p}(T) \mathrm{d} T ; \Delta S=\int \Delta C_{p}(T) / T \mathrm{~d} T$, and the Gibbs equation, $\Delta G_{(T)}^{\circ}=\Delta H-T \Delta S$; where $\Delta C_{p}$ is the anomalous heat capacity of the ODN solution during the unfolding process, $\Delta H$ and $\Delta S$ are the unfolding enthalpy and entropy, respectively, assumed to be temperature-independent. $\Delta G_{(\mathrm{T})}^{\circ}$ is the free energy at a temperature $T$.

\section{Isothermal titration calorimetry (ITC)}

The heat for the reaction of a pseudoknot with its complementary strand was measured directly by isothermal titration calorimetry using the ITC 200 from GE Microcal (Northampton, MA). A $40 \mu \mathrm{L}$ syringe was used to inject the titrant; mixing was effected by stirring this syringe at $1000 \mathrm{rpm}$. Typically, we used 5-7 injections of $2 \mu \mathrm{L}$ of pseudoknot solution with at least 2-fold lower concentration than the solution of the complementary strand in the cell, and over a time of 4-8 minutes between injections. The reaction heat of each injection is measured by integration of the area of the injection curve, corrected for the dilution heat of the titrant, and normalized by the moles of titrant added to yield the reaction enthalpy, $\Delta H_{\text {ITC }}[27-29,42]$. All titrations ITC experiments were designed to obtain the heat, $\Delta H_{\mathrm{ITC}}$ for each targeting reaction by averaging the reaction heat of at least five injections under unsaturated conditions. These $\Delta H_{\mathrm{ITC}}$ terms correspond to the formation of duplex products. To determine the free energy, $\Delta G_{\mathrm{ITC}}$ for each targeting reaction, we use the following relationship, $\Delta G_{\text {IIC }}=$
$\Delta G_{\mathrm{HC}}\left(\Delta H_{\mathrm{ITC}} / \Delta H_{\mathrm{HC}}\right)$ [27-29], while the Gibbs equation is used to determine the $T \Delta S_{\text {ITC }}$ parameter, where $T$ is the temperature of the ITC experiments.

\section{Overall experimental protocol}

We used initially UV melting techniques to characterize the helix-coil transition of each molecule as a function of strand concentration. DSC experiments are carried out to determine $T_{\mathrm{M}} \mathrm{s}$ and unfolding thermodynamic profiles for each pseudoknot and for the other reactants and products of each targeting reaction $[\mathbf{4 0 , 4 3 ]}$. The DSC data is used to set up Hess cycles to yield thermodynamic profiles for these reactions. Then, ITC titrations are used to measure directly the heat $\left(\Delta H_{\text {ITC }}\right)$ of each targeting reaction, which are compared with the Hess cycle data [27-29].

\section{Results and discussion \\ Unfolding thermodynamics of pseudoknots}

Figure 2a shows typical UV melting curves for the helix-coil transition of each pseudoknot, their sigmoidal behavior is characteristic of the temperature-induced unfolding of a nucleic acid oligonucleotide. The $T_{M}$ dependences on strand concentration are shown in Figure $\mathbf{2} \mathbf{b}$, the $T_{M}$ remains constant for each pseudoknot, indicative of their intramolecular formation at low temperatures. The DSC curves of each pseudoknot are shown in Figure $2 \mathrm{c}$ and the resulting thermodynamic profiles are shown in Table 1. Each curve shows slightly asymmetric peak, which is not consistent with the unfolding of an intramolecular complex. This observation may be explained in terms of loop constraints on the pseudoknot stems, which reduces favorable stacking contributions. The overall effect is most noticeable with PsK-5, which actually shows a small shoulder at around $37^{\circ} \mathrm{C}$. We obtained unfolding enthalpies of $60.1 \mathrm{kcal} / \mathrm{mol}$ (PsK-5) and $87.5 \mathrm{kcal} / \mathrm{mol}$ (PsK-9), while an unfolding enthalpy of $69.4 \mathrm{kcal} . \mathrm{mol}$ (data not shown) was obtained for a duplex (5'-TCTCTTTTTTTTT/5'-TTAAAAAAAAGAGATT) with sequence similar to their stem and with two thymines flanking each end. 

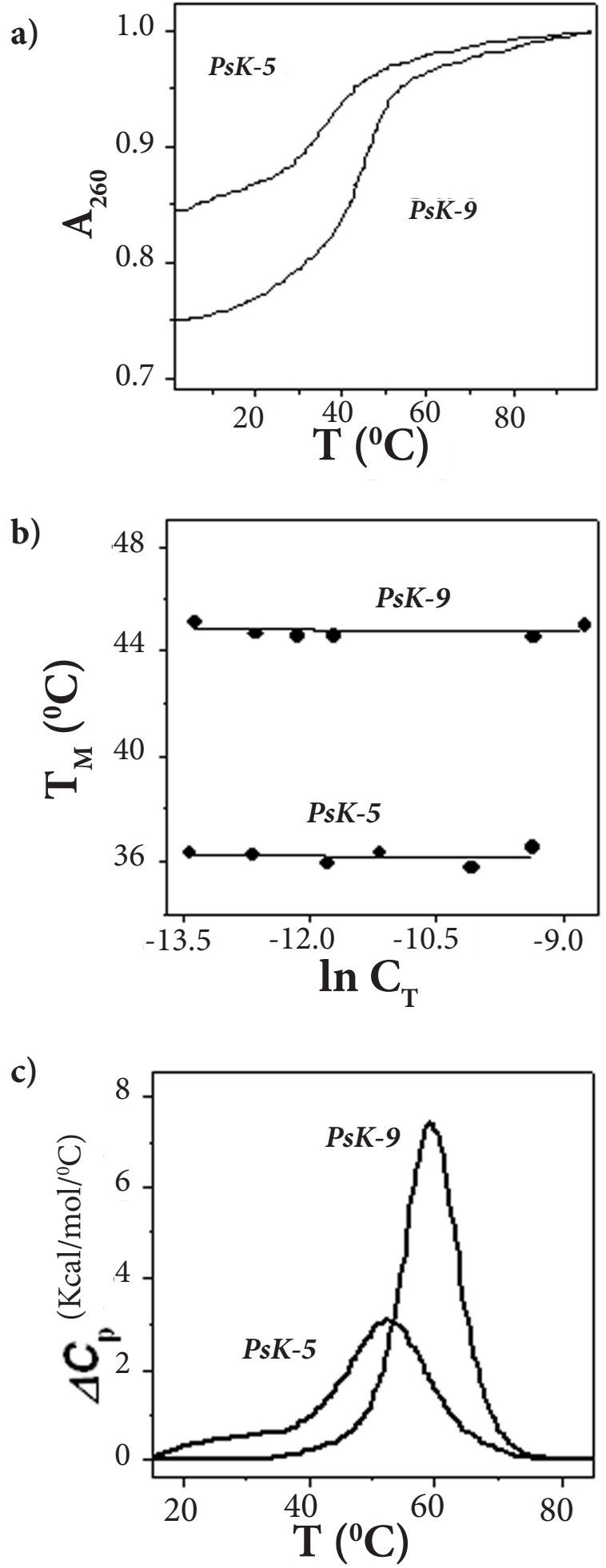

Figure 2. Melting curves for pseudoknots. a) UV curves showing concentration independence for PsK-5 and PsK-9 b) $\ln C_{T}$ plots to determine concentration dependence on $T_{M}$ c) DSC curves for PsK-5 (0.126 mM) and PsK-9 (0.120 mM). UV experiments were carried out in $10 \mathrm{mM} \mathrm{NaPi}$ and DSC experiments were carried out in $10 \mathrm{mM} \mathrm{NaPi}, 100 \mathrm{mM} \mathrm{NaCl}$ buffer at pH 7.0.
Table 1. Unfolding and targeting thermodynamic profiles.

\begin{tabular}{|c|c|c|c|c|c|}
\hline & Transition & $\begin{array}{l}T_{\mathrm{M}} \\
\left({ }^{\circ} \mathrm{C}\right)\end{array}$ & $\begin{array}{l}\Delta H^{\circ} \\
(\mathrm{kcal} / \mathrm{mol})\end{array}$ & $\begin{array}{l}T \Delta S \\
(\mathrm{kcal} / \mathrm{mol})\end{array}$ & $\begin{array}{l}\Delta \boldsymbol{G}_{(5)}^{\circ} \\
(\mathrm{kcal} / \mathrm{mol})\end{array}$ \\
\hline PsK-5 & -- & 52.8 & 60.1 & 51.6 & 8.5 \\
\hline$C S-5$ & -- & 47.9 & 38.4 & 33.3 & 5.1 \\
\hline PsKDup-5 & -- & 49.6 & 135 & 116 & 18.7 \\
\hline PsK-9 & -- & 59.3 & 87.5 & 73.3 & 14.2 \\
\hline CS-9 & -- & 48.6 & 48.5 & 41.9 & 6.6 \\
\hline \multirow[t]{5}{*}{ PsKDup-9 } & $1^{\text {st }}$ & 47.7 & 59.2 & 51.3 & 7.9 \\
\hline & $2^{\text {nd }}$ & 58.3 & 102 & 85.6 & 16.4 \\
\hline & Total & -- & 161 & 137 & 24.3 \\
\hline & DSC & & & ITC & \\
\hline & $\underset{(\mathrm{kcal} / \mathrm{mol})}{\Delta \boldsymbol{H}_{\mathrm{HC}}}$ & $\begin{array}{l}\Delta \boldsymbol{G}_{\mathrm{HC}}^{\circ} \\
(\mathrm{kcal} / \mathrm{mol})\end{array}$ & $\begin{array}{l}T \Delta \boldsymbol{S}_{\mathrm{HC}} \\
(\mathrm{kcal} / \mathrm{mol})\end{array}$ & $\begin{array}{l}\Delta \boldsymbol{H}_{\mathrm{rTC}} \\
(\mathrm{kcal} / \mathrm{mol})\end{array}$ & $\begin{array}{l}\Delta \boldsymbol{G}_{\mathrm{ITC}}^{\circ} \\
(\mathrm{kcal} / \mathrm{mol})\end{array}$ \\
\hline PsKDup-5 & -36.5 & -5.1 & -31.4 & -28.2 & -3.9 \\
\hline PsKDup-9 & -25.0 & -3.5 & -21.7 & -23.7 & -3.2 \\
\hline
\end{tabular}

All experiments were done in $10 \mathrm{mM}$ sodium phosphate buffer and $100 \mathrm{mM} \mathrm{NaCl}$ at $\mathrm{pH}$ 7.0. Experimental errors are as follows: $T_{\mathrm{M}}( \pm$ $\left.0.5^{\circ} \mathrm{C}\right), \Delta \mathrm{H}( \pm 5 \%), T \Delta S( \pm 5 \%), \Delta G_{(5)}^{0}( \pm 7 \%), \Delta H_{\mathrm{ITC}}( \pm 5 \%)$,

$\Delta G_{\text {ITC }}^{\circ}( \pm 7 \%), \Delta H_{\mathrm{HC}}( \pm 10 \%), T \Delta S_{\mathrm{HC}}^{(5)}( \pm 10 \%), \Delta G_{\mathrm{HC}}^{0}( \pm 14 \%)$.

Thermodynamic profiles for the unfolding of the putative structures and their targeting single strands are included.

This enthalpy comparison shows that the loops of PsK-5 are constrained while the longer loop of $\boldsymbol{P} \boldsymbol{S K} \mathbf{- 9}$ is actually releasing this tension. However, the main observation is PsK-9 unfolds with a higher $T_{\mathrm{M}^{\prime}}$ by $6.5^{\circ} \mathrm{C}$, and higher unfolding enthalpy, by $27.4 \mathrm{kcal} / \mathrm{mol}$ (Table 1). This indicates that $\boldsymbol{P s} \mathbf{K}-\mathbf{9}$ is more stable with improved base-pair stacks. Alternatively, the observed additional heat suggests that the 9 thymine loop of PsK-9 is located in the ceiling of the major groove of its $7 \mathrm{~A} \cdot \mathrm{T}$ stem, and three thymines (starred thymines of Figure 1 ) are involved in the formation of three $T^{*} A \cdot T$ base-triplets (or two $T^{*} A T / T^{*} A T$ base-triplet stacks) $[44,45]$. Overall, the unfolding of each pseudoknot takes place through the typical unfavorable enthalpy-favorable entropy compensation. Unfavorable enthalpy contributions correspond to energy needed to break base pairing and base-pair stacking interactions, while favorable entropy contributions correspond to the higher disorder state of the single strand at high temperature and the putative release of ions and water molecules [27-29,46]. In summary, the folding of PsK-9 is thermodynamically more stable than PsK-5 (reverse signs of Table 1), by $-5.7 \mathrm{kcal} / \mathrm{mol}$, resulting in a more compact molecule.

Targeting of pseudoknots with complementary strands To confirm PsK-9 is forming a more compact pseudoknot, we investigated the reaction of each pseudoknot with its corresponding complementary strand to form duplex products with dangling ends: 
a) PsK-5+CS-5 $\rightleftharpoons$ PsKDup-5; and

b) PsK-9+CS-9 $\rightleftharpoons$ PsKDup-9

Each reaction was investigated in two ways: a) indirectly by determining unfolding thermodynamic profiles for the reactants and products of each targeting reaction, and b) directly using ITC techniques.

Unfolding thermodynamics for the species of each targeting reaction

Figure 3 shows the DSC thermograms of each pseudoknot, complementary strand, and duplex product, and Table 1 has the resulting thermodynamic profiles from the analysis of these thermograms. The DSC of each pseudoknot was discussed earlier; their unfolding curves are included in this figure for clarity and for explaining the unfolding of each product duplex.

a)

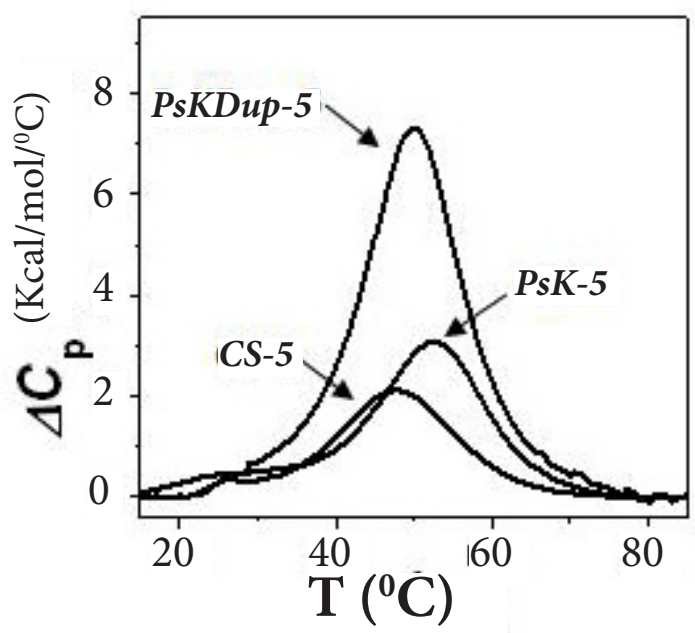

b)

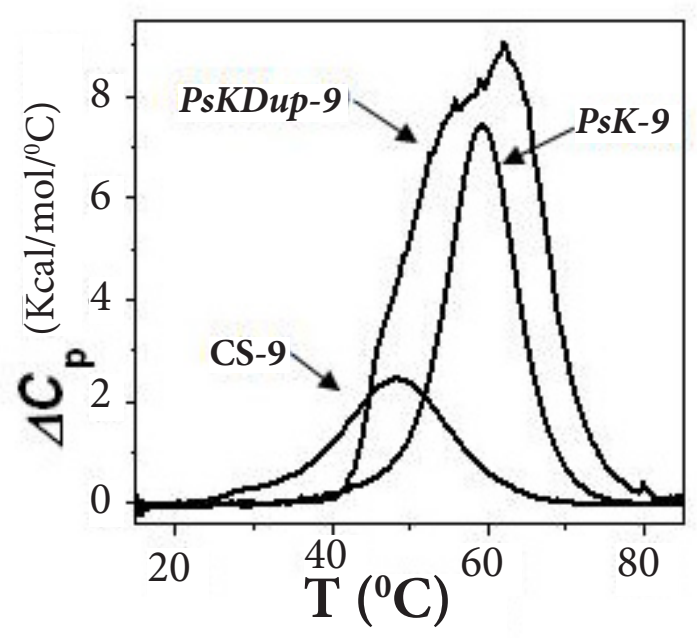

Figure 3. DSC curves for pseudoknots, complementary strands and duplexes. a) DSC curves of $\boldsymbol{P s} \boldsymbol{K}-\mathbf{5}(0.126 \mathrm{mM})$, CS-5 (0.182 mM) and PsKDup-5 (0.039 mM) b) DSC curves for PsK-9 (0.120 mM), CS-9 (0.136 mM) and PsKDup-9 $(0.025 \mathrm{mM})$. All experiments were carried out in $10 \mathrm{mM} \mathrm{NaPi}$, $100 \mathrm{mM} \mathrm{NaCl}$ at $\mathrm{pH} 7.0$.
The DSC of each single strand shows monophasic transitions with $T_{\mathrm{M}} \mathrm{s}$ and $\Delta H \mathrm{~s}$ of $47.9^{\circ} \mathrm{C}, 38.4 \mathrm{kcal} / \mathrm{mol}(\mathrm{CS}-5)$ and $48.6^{\circ} \mathrm{C}$, $48.5 \mathrm{kcal} / \mathrm{mol}$ (CS-9), respectively. UV melting experiments as a function of strand concentration (data not shown) show their $T_{\mathrm{M}} \mathrm{s}$ remain constant, indicating their intramolecular formation. Based on their sequence, magnitude of their enthalpies, and assuming an enthalpy of 7-8 kcal per mol of base-pair stack, these single strands are forming hairpin loops with dangling ends and with 5 (CS-5) and 6 (CS-9) base-pair stacks in their stems.

UV melting experiments of the duplex products, as a function of strand concentration, show that the $T_{\mathrm{M}} \mathrm{s}$ increased slightly (data not shown), indicative of their bimolecular formation. These $T_{\mathrm{M}}$-dependences are consistent with the unfolding of duplexes with 19 and 23 base pairs, which are approaching the unfolding behavior of polymers. The DSC curves of each product duplex (Figure 3) show PsKDup-5 unfolds in an apparent monophasic transition with a $T_{M}$ of $49.6^{\circ} \mathrm{C}$ and $\Delta H$ of $135 \mathrm{kcal} / \mathrm{mol}$, while PsKDup-9 unfolds in a biphasic transition with $T_{\mathrm{M}} \mathrm{s}$ of $47.7^{\circ} \mathrm{C}$ and $58.3^{\circ} \mathrm{C}$ and total $\Delta H$ of 161 $\mathrm{kcal} / \mathrm{mol}$. Each DSC profile corresponds to the unfolding of the duplex followed by the folding and sequential unfolding of the corresponding pseudoknot and hairpin (single strands). For instance, the monophasic unfolding of PsKDup-5 is due to the similar $T_{M} \mathrm{~S}$ of the structures formed by the reactants and product of this reaction, which are within $5^{\circ} \mathrm{C}$. On the other hand, PsKDup-9 shows a biphasic transition (Figure $3 \mathbf{b}$ ), the first transition corresponds to the unfolding of the duplex into partial folded of both PsK-9 and CS-9, followed by the simultaneous unfolding of CS-9 and pseudoknot. The higher $\Delta H$ term of PsKDup-9, by $26 \mathrm{kcal} / \mathrm{mol}$, corresponds to the formation of two additional base-triplet stacks.

We created Hess cycles with this unfolding data to generate indirectly thermodynamic profiles for each targeting reaction i.e., we added the thermodynamic profiles of the pseudoknot and single strand (hairpin), and subtracted the thermodynamic profiles of the duplex. The resulting data is shown in the last two entries of Table 1. This exercise yielded $\Delta G_{\mathrm{HC}}^{\circ}$ and $\Delta H_{\mathrm{HC}}$ of $-5.1 \mathrm{kcal} / \mathrm{mol} \&-37 \mathrm{kcal} / \mathrm{mol}$ (PsK-5), and $-3.5 \mathrm{kcal} / \mathrm{mol} \&-25$ $\mathrm{kcal} / \mathrm{mol}(\boldsymbol{P} \boldsymbol{s} \boldsymbol{K}-\mathbf{9})$, respectively. Both reactions are favorable and enthalpy driven. However, the targeting of PsK-9 is less favorable, which is consistent with the higher stability of this pseudoknot. Furthermore, we obtained unfavorable $T \Delta S_{H C}$ terms of -31.4 (PsK-5), and $-21.7 \mathrm{kcal} / \mathrm{mol}$ (PsK-9), which correspond to the net uptake of ions and water molecules by the duplex products of each reaction, since the conformational entropy change is considered similar for each reaction.

Targeting reactions measured directly by ITC techniques The heat for each targeting reaction was measured by ITC under unsaturated conditions, using ODN concentrations and temperatures that guaranteed $100 \%$ formation of the final duplex products. ITC titrations were carried out at $5^{\circ} \mathrm{C}$, the heat of each injection was corrected for its dilution heat and 
Reiling et al. Biochemical Compounds 2014,

normalized by the concentration of the limiting reagent to yield reaction enthalpies, $\Delta H_{\text {ITC }}$. The ITC titrations are shown in Figure 4a (PsK-5) and Figure 4b (PsK-9), the shape of these curves show that the initial enthalpies are more exothermic, $-32.0 \mathrm{kcal} / \mathrm{mol}$ and $-33 \mathrm{kcal} / \mathrm{mol}$, and gradually reaching a plateau at $-27.3 .0 \mathrm{kcal} / \mathrm{mol}$ and $-21.2 \mathrm{kcal} / \mathrm{mol}$, respectively. The net exothermicity of these enthalpy values corresponds to a complete override of the endothermic heat contributions (disruption of the base-pair stacks of both pseudoknot and hairpin loop) by the exothermic heat contributions (formation of base-pair stacks of the duplex product). An extra exothermic/endothermic term should be included due to hydration changes from the participating reaction species, which may be accounted for the variability of the reaction enthalpies in these titrations. The average heat for all injections of a particular titration are $\Delta H_{\mathrm{ITC}} \mathrm{S}$ of $-28.2 \pm 1.4$ $\mathrm{kcal} / \mathrm{mol}(\boldsymbol{P} \boldsymbol{s}$ K-5) ) and $-23.7 \pm 1.2 \mathrm{kcal} / \mathrm{mol}$ (PsK-9), which are in good agreement with the $\Delta H_{\mathrm{HC}}$ values of $-36.5 \pm 3.7 \mathrm{kcal} / \mathrm{mol}$ and $-25.0 \pm 2.5 \mathrm{kcal} / \mathrm{mol}$, respectively, obtained indirectly by the Hess cycles from the DSC data.

The $\Delta G^{\circ}{ }_{I C}$ at $5{ }^{\circ} \mathrm{C}$ for each targeting reaction were obtained from the $\Delta G^{\circ}{ }_{\mathrm{HC}}$ values by using a temperature factor $\left(=\Delta H_{\mathrm{ITC}}\right.$ ' $\Delta H_{\mathrm{HC}}$, which assumes $\Delta H_{\mathrm{HC}}$ to be independent of temperature i.e., $\Delta C p=0$. The $T \Delta S_{\text {ITC }}$ parameters were calculated using the Gibbs equation. The overall results are shown in Table 1. We obtained favorable $\Delta G^{\circ}{ }_{\text {ITC }}$ contributions for each targeting reaction, each complementary strand is able to invade and disrupt the pseudoknot structure. However, the reaction for the targeting of $\boldsymbol{P s} \mathbf{K}-\mathbf{9}$ is less favorable in spite of forming a more stable duplex, by $0.7 \mathrm{kcal} / \mathrm{mol}$. This result is consistent with the higher stability PsK-9 and confirms the formation of a local triplex in the stem of PsK-9 consistent of three $T^{*} A \cdot T$ base-triplets (or two T*AT/T*AT base-triplet stacks).

\section{Conclusions}

We have investigated the thermodynamic stability of two pseudoknots to determine the formation of a local and short triplex in the pseudoknot with a longer thymine loop. Specifically, we used a combination of UV, DSC, and ITC techniques to determine the unfolding thermodynamics of a pair of pseudoknots and their reaction with complementary strands. The favorable folding of DNA pseudoknots results from the typical favorable enthalpy-unfavorable entropy compensation, confirming the flexibility of DNA strands being able to form pseudoknots that can be used to mimic known RNA secondary structures. The folding data shows that $\boldsymbol{P} \boldsymbol{s} \mathbf{K}-\mathbf{9}$ is more stable due to a more favorable enthalpy. This enthalpy value corresponds to the partial folding of the thymine loop (third strand) on the major groove of the duplex, yielding a net formation of two $T^{*} A T / T^{*} A T$ base-triplet stacks at the middle of its stem. The targeting thermodynamic data indicated that each complementary strand is able to disrupt the pseudoknots. However, the disruption of PsK-9 takes place with a less favorable free energy contribution, confirming the a)

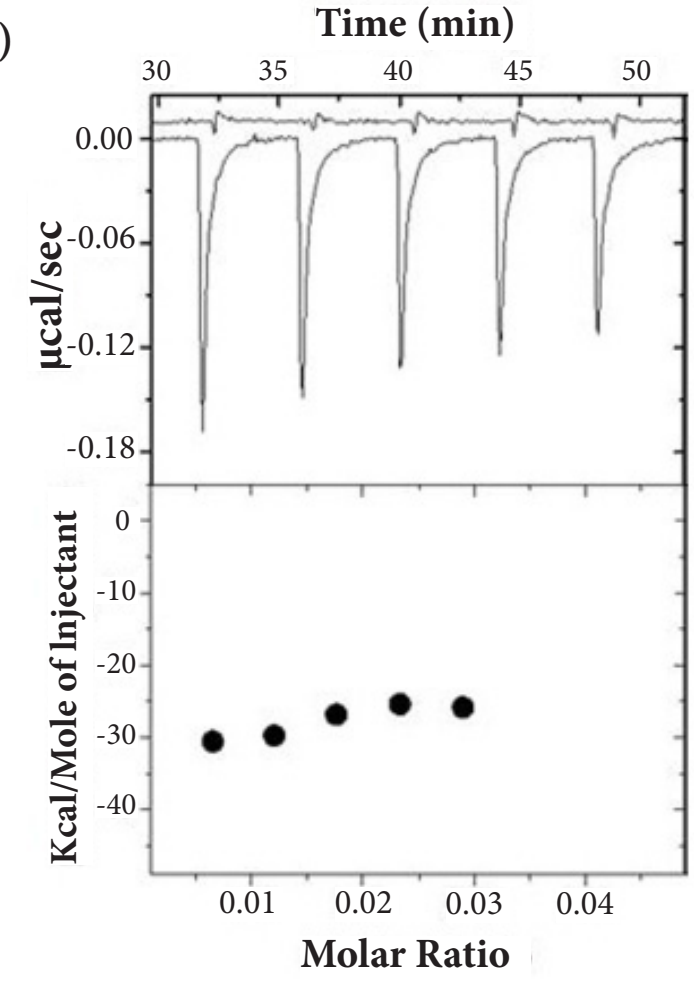

b)

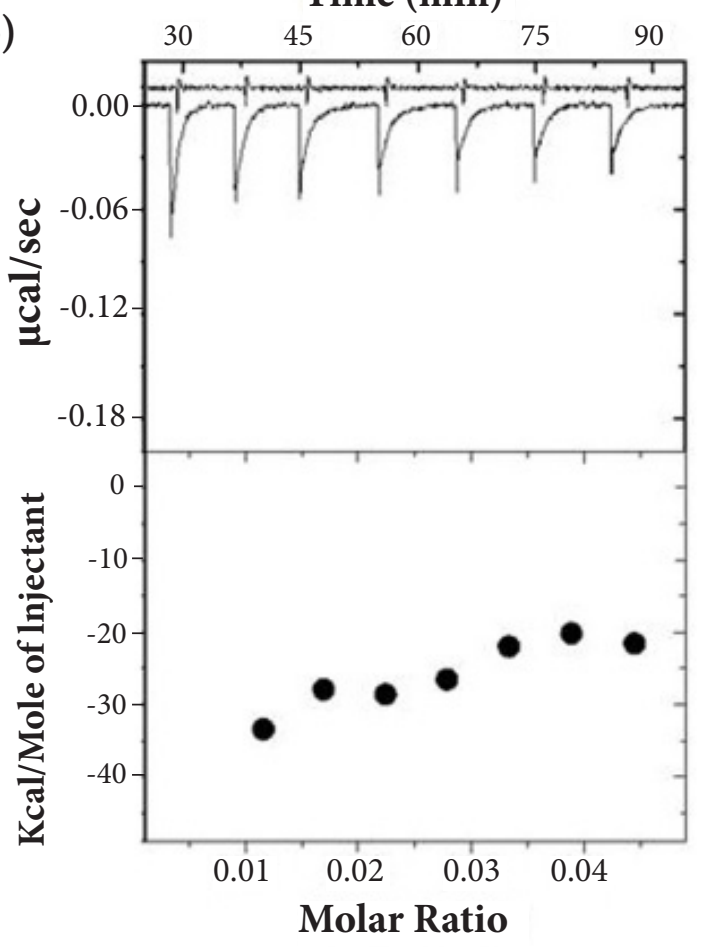

Figure 4. Targeting reactions. a) ITC injections of PsK-5 (0.065 $\mathrm{mM}$ ) into $\boldsymbol{C S}-5$ (0.123 $\mathrm{mM})$. Also shown are the dilution heats of PsK-5 into buffer. b) ITC injections of PsK-9 (0.067 mM) into CS-9 $(0.128 \mathrm{mM})$. Also shown are the dilution heats of PsK-9 into buffer. All experiments performed in $10 \mathrm{mM} \mathrm{NaPi}$, $100 \mathrm{mM} \mathrm{NaCl}$ at $\mathrm{pH} 7.0$. 
Reiling et al. Biochemical Compounds 2014,

formation of a short and local triplex.

The main observation from this study is that a triplex is able to form in the pseudoknot if the loop length and sequence are appropriate. The favorable targeting of these pseudoknots depends on the length and sequence of the complementary strand. However, the favorable free energy term of these targeting reactions may well be increased by improving the stability of the duplex products, by using longer single strands with complementary sequences and/or DNA intramolecular structures with loops containing a larger number of un-paired bases. In general, the higher the number of base-pairs and base-pair stacks that are formed in the duplex product, the higher the free energy term; specifically, if a larger number of unpaired bases are involved in this base-pairing. This investigation of the targeting of DNA pseudoknots has enabled us to improve our method, based on physicochemical principles, to determine the thermodynamics of the targeting of nucleic acid secondary structures that can be used to control the expression of genes.

\section{Competing interests}

The authors declare that they have no competing interests. Authors' contributions

\begin{tabular}{|l|c|c|}
\hline Authors' contributions & CR & LAM \\
\hline Research concept and design & -- & $\checkmark$ \\
\hline Collection and/or assembly of data & $\checkmark$ & -- \\
\hline Data analysis and interpretation & $\checkmark$ & $\checkmark$ \\
\hline Writing the article & $\checkmark$ & $\checkmark$ \\
\hline Critical revision of the article & $\checkmark$ & $\checkmark$ \\
\hline Final approval of article & $\checkmark$ & $\checkmark$ \\
\hline Statistical analysis & $\checkmark$ & $\checkmark$ \\
\hline
\end{tabular}

\section{Acknowledgement}

This work was supported by National Science Foundation Grant MCB-1122029 and GAANN grant P200A120231 (C.R.) from the U.S. Department of Education.

Publication history

Editor: Leonid Breydo, University of South Florida, USA.

EIC: John Wade, University of Melbourne, Australia.

Received: 17-Jun-2014 Final Revised: 01-Aug-2014

Accepted: 07-Aug-2014 Published: 28-Aug-2014

\section{References}

1. Rich A. DNA comes in many forms. Gene. 1993; 135:99-109. | Article | PubMed

2. Gehring K, Leroy JL and Gueron M. A tetrameric DNA structure with protonated cytosine.cytosine base pairs. Nature. 1993; 363:561-5. | Article | PubMed

3. Kaushik M, Suehl N and Marky LA. Calorimetric unfolding of the bimolecular and i-motif complexes of the human telomere complementary strand, d(C(3)TA(2))(4). Biophys Chem. 2007; 126:15464. | Article | PubMed

4. Juliano RL, Astriab-Fisher A and Falke D. Macromolecular therapeutics: emerging strategies for drug discovery in the postgenome era. $\mathrm{Mol}$ Interv. 2001; 1:40-53. | PubMed

5. Crooke ST. Molecular mechanisms of action of antisense drugs. Biochim

\section{Biophys Acta. 1999; 1489:31-44. | Article | PubMed}

6. Helene $C$. Rational design of sequence-specific oncogene inhibitors based on antisense and antigene oligonucleotides. Eur J Cancer. 1991; 27:1466-71. | Article | PubMed

7. Helene $C$. Control of oncogene expression by antisense nucleic acids. Eur J Cancer. 1994; 30A:1721-6. | PubMed

8. Firulli $A B$, Maibenco $D C$ and Kinniburgh AJ. Triplex forming ability of a c-myc promoter element predicts promoter strength. Arch Biochem Biophys. 1994; 310:236-42. | Article | PubMed

9. Fox KR. Long (dA)n $x(d T) n$ tracts can form intramolecular triplexes under superhelical stress. Nucleic Acids Res. 1990; 18:5387-5391.

10. Han $\mathrm{H}$ and Hurley LH. G-quadruplex DNA: a potential target for anticancer drug design. Trends Pharmacol Sci. 2000; 21:136-42. | Article | PubMed

11. Mills $\mathrm{M}$, Lacroix $\mathrm{L}$, Arimondo PB, Leroy $\mathrm{JL}$, Francois $\mathrm{JC}$, Klump $\mathrm{H}$ and Mergny JL. Unusual DNA conformations: implications for telomeres. Curr Med Chem Anticancer Agents. 2002; 2:627-44. | Article | PubMed

12. Bock LC, Griffin LC, Latham JA, Vermaas EH and Toole JJ. Selection of single-stranded DNA molecules that bind and inhibit human thrombin. Nature. 1992; 355:564-6. | Article | PubMed

13. Wang KY, Krawczyk SH, Bischofberger N, Swaminathan S and Bolton PH. The tertiary structure of a DNA aptamer which binds to and inhibits thrombin determines activity. Biochemistry. 1993; 32:11285-11292. | Article

14. Rando RF, Ojwang J, Elbaggari A, Reyes GR, Tinder R, McGrath MS and Hogan ME. Suppression of human immunodeficiency virus type 1 activity in vitro by oligonucleotides which form intramolecular tetrads. $J$ Biol Chem. 1995; 270:1754-60. | Article | PubMed

15. Rastogi T, Beattie TL, Olive JE and Collins RA. A long-range pseudoknot is required for activity of the Neurospora VS ribozyme. EMBO J. 1996; 15:2820-2825

16. Ke A, Zhou K, Ding F, Cate JH and Doudna JA. A conformational switch controls hepatitis delta virus ribozyme catalysis. Nature. 2004; 429:2015. | Article | PubMed

17. Isambert $\mathrm{H}$ and Siggia ED. Modeling RNA folding paths with pseudoknots: application to hepatitis delta virus ribozyme. Proc Natl Acad Sci U S A. 2000; 97:6515-20. | Article | PubMed Abstract | PubMed Full Text

18. Adams PL, Stahley MR, Kosek AB, Wang J and Strobel SA. Crystal structure of a self-splicing group I intron with both exons. Nature. 2004; 430:45-50. | Article | PubMed

19. Huard $\mathrm{S}$ and Autexier $\mathrm{C}$. Targeting human telomerase in cancer therapy. Curr Med Chem Anticancer Agents. 2002; 2:577-87. | Article I PubMed

20. Theimer CA, Blois CA and Feigon J. Structure of the human telomerase RNA pseudoknot reveals conserved tertiary interactions essential for function. Mol Cell. 2005; 17:671-82. | Article | PubMed

21. Souliere MF, Altman RB, Schwarz V, Haller A, Blanchard SC and Micura $R$. Tuning a riboswitch response through structural extension of a pseudoknot. Proc Natl Acad Sci U S A. 2013; 110:E3256-64. | Article | PubMed Abstract | PubMed Full Text

22. Huang $X$, Yang $Y$, Wang $G$, Cheng $Q$ and Du Z. Highly conserved RNA pseudoknots at gag-pol junction of HIV-1 suggest a novel mechanism for -1 ribosomal frameshifting. RNA. 2014; 20:587-93.

23. Chen G, Chang KY, Chou MY, Bustamante $C$ and Tinoco I, Jr. Triplex structures in an RNA pseudoknot enhance mechanical stability and increase effieciency of $\mathbf{- 1}$ ribosomal frameshifting. PNAS. 2009; 106:12706-12711.

24. Beal PA and Dervan PB. Second structural motif for recognition of DNA by oligonucleotide-directed triple-helix formation. Science. 1991; 251:1360-3. | Article | PubMed

25. Zahler AM, Williamson JR, Cech TR and Prescott DM. Inhibition of telomerase by G-quartet DNA structures. Nature. 1991; 350:718-20. | Article | PubMed

26. Folini M, Pennati $\mathrm{M}$ and Zaffaroni $\mathrm{N}$. Targeting human telomerase by antisense oligonucleotides and ribozymes. Curr Med Chem Anticancer 
Reiling et al. Biochemical Compounds 2014,

Agents. 2002; 2:605-12. | PubMed

27. Lee HT, Olsen CM, Waters L, Sukup H and Marky LA. Thermodynamic contributions of the reactions of DNA intramolecular structures with their complementary strands. Biochimie. 2008; 90:1052-63. | Article | PubMed

28. Lee HT, Carr C, Siebler H, Waters L, Khutsishvili I, Iseka F, Domack B, Olsen CM and Marky LA. A thermodynamic approach for the targeting of nucleic acid structures using their complementary single strands. Methods Enzymol. 2011; 492:1-26. | Article | PubMed

29. Khutsishvili I, Johnson SE, Reiling C, Prislan I, Lee HT and Marky LA. Interaction of DNA intramolecular structures with their complementary strands: a thermodynamic approach for the control of gene expression. In: VA Erdmann et al. (Eds.), Chemical Biology of Nucleic Acids, RNA Technologies 2014; 367-383. | Article

30. Mahato RI, Cheng K and Guntaka RV. Modulation of gene expression by antisense and antigene oligodeoxynucleotides and small interfering RNA. Expert Opin Drug Deliv. 2005; 2:3-28. | Article | PubMed

31. Soyfer VN and Potaman VN. Triple-helical Nucleic Acids. Springer. 1996; 360

32. Maher $L$, 3rd, Wold B and Dervan PB. Inhibition of DNA binding proteins by oligonucleotide-directed triple helix formation. Science. 1989; 245:725-30. | Article | PubMed

33. Fox KR. Targeting DNA with triplexes. Curr Med Chem. 2000; 7:17-37. | Article | PubMed

34. Brown PM, Madden CA and Fox KR. Triple-helix formation at different positions on nucleosomal DNA. Biochemistry. 1998; 37:16139-16151. | Article

35. Vasquez KM , Dagle JM, Weeks DL and Glazer PM. Chromosome targeting at short polypurine sites by cationic triplex-forming oligonucleotides. J. Biol. Chem. 2001; 276:38536-38541. | Article

36. Breslauer KJ, Frank R, Blocker $\mathrm{H}$ and Marky LA. Predicting DNA duplex stability from the base sequence. Proc Natl Acad Sci U S A. 1986; 83:3746-50. | Article | PubMed Abstract | PubMed Full Text

37. Sugimoto N, Nakano S, Katoh M, Matsumura A, Nakamuta H, Ohmichi $\mathrm{T}$, Yoneyama $\mathrm{M}$ and Sasaki $\mathrm{M}$. Thermodynamic parameters to predict stability of RNA/DNA hybrid duplexes. Biochemistry. 1995; 34:11211-6. | Article | PubMed

38. SantaLucia J, Jr., Allawi HT and Seneviratne PA. Improved nearestneighbor parameters for predicting DNA duplex stability. Biochemistry. 1996; 35:3555-62. | Article | PubMed

39. Borer PN. Optical properties of nucleic acids, absorptions and circular dichroism spectra. In: Fasman GD (Eds.) Handbook of Biochemistry and Molecular Biology, $3^{\text {rd }}$ Edition. 1975; 589-595.

40. Marky LA and Breslauer KJ. Calculating thermodynamic data for transitions of any molecularity from equilibrium melting curves. Biopolymers. 1987; 26:1601-20. | Article | PubMed

41. Marky LA, Maiti S, Olsen CM, Shikiya R, Johnson SE, Kaushik M and Khutsishvili I. Building blocks of nucleic acid nanostructures: unfolding thermodynamics of intramolecular DNA complexes. In V. Labhasetwar, D. Leslie-Pelecky (Eds.), Biomedical Applications of Nanotechnology 2007; 191-225. | Article

42. Wiseman T, Williston S, Brandts JF and Lin LN. Rapid measurement of binding constants and heats of binding using a new titration calorimeter. Anal Biochem. 1989; 179:131-7. | Article | PubMed

43. Maiti S, Kankia B, Khutsishvili I and Marky LA. Melting behavior and ligand binding of DNA intramolecular secondary structures. Biophys Chem. 2011; 159:162-71. | Article | PubMed

44. Soto AM, Rentzeperis D, Shikiya R, Alonso M and Marky LA. DNA intramolecular triplexes containing dT --> dU substitutions: unfolding energetics and ligand binding. Biochemistry. 2006; 45:3051-9. | Article | PubMed

45. Soto AM, Loo J and Marky LA. Energetic contributions for the formation of TAT/TAT, TAT/CGC(+), and CGC(+)/CGC(+) base triplet stacks. J Am Chem Soc. 2002; 124:14355-63. | Article | PubMed

46. Marky LA and Kupke DW. Enthalpy-entropy compensations in nucleic acids: contributions of electrostriction and structural hydration.
Methods Enzymol. 2000; 323:419-441. | Article | PubMed

Citation:

Reiling C and Marky LA. Contributions of the loops on the stability and targeting of DNA pseudoknots. Bio Chem Comp. 2014; 2:3. http://dx.doi.org/10.7243/2052-9341-2-3 